\title{
La Psicología Experimental en dos universidades paraguayas*
}

\section{The experimental Psychology in two paraguayan universities}

\begin{abstract}
José E. García**
Universidad de la Integración de las Américas, Asunción

Universidad Católica, Sede Regional

Itapúa, Encarnación, Paraguay
\end{abstract}

Recibido: 24 de marzo de 2009

Revisado: 29 de mayo de 2009

Aceptado: 30 de octubre de 2009

\section{Resumen}

Varias de las carreras de Psicología que actualmente funcionan en Paraguay incluyen cátedras de Psicología Experimental en sus mallas curriculares. Uno de los objetivos de estas cátedras es la aplicación del método de la experimentación para elaborar trabajos de investigación de sus estudiantes, con lo que cumplen el doble propósito del aprendizaje didáctico y el desarrollo de conocimiento nuevo. Sin embargo, en Paraguay estas cátedras deben realizarse en medio de una escasez muy aguda de recursos e infraestructura. Este trabajo discute de manera crítica tales problemas. En primer lugar, se hace una exposición sobre los antecedentes de la Psicología Experimental en el Paraguay. Luego, se detalla la producción de los estudiantes en dos universidades haciendo especial referencia a los temas que abordan las investigaciones y el tipo de estadística utilizada. Los trabajos fueron completados durante el segundo semestre de los años 2006, 2007 y 2008 en la Universidad de la Integración de las Américas (UNIDA), ubicada en Asunción (2006) y la Universidad Católica, sede Regional Itapúa (UCI) en la ciudad de Encarnación (2006, 2007 y 2008). En la sección final del trabajo se identifican y discuten algunos de los problemas principales que

Artículo de investigación no financiado institucionalmente.

Correspondencia: José E. García, Casilla de Correo 1839, Asunción, Paraguay. Correo electrónico: joseemiliogarcia@hotmail.com 
dificultan la investigación psicológica en las universidades paraguayas con especial énfasis en la aplicación del método experimental.

Palabras clave: Psicología Experimental, método experimental, psicología en Paraguay, investigación psicológica.

\section{Abstract}

The production of psychological knowledge that is both original and high level can be considered the main condition to place psychology as a field that takes part in the general concert of the sciences. In Paraguay several of its psychological departments has courses of Experimental Psychology as a part of their academic platform. One of the regular objectives for these courses is the application of the experimental method in the student research works, with the double purpose of both didactical learning and the developing of new knowledge. However, in Paraguay these courses carry out their teaching activities in the middle of a great scarcity both of resources and infrastructure. But some universities bear their basic reserch purposes to a final stage. This article discusses such problems in a critical way. First, a general exposition on the background of experimental psychology in Paraguay can be found. Second, the article details the students' work with special reference to both the subject of research and the used statistics. The papers were completed during the second semester of the years 2006, 2007 and 2008 at the University of the Integration of the Americas (UNIDA) located in the city of Asunción (2006) and the Catholic University, Regional See of Itapúa (UCl) in the city of Encarnación (2006, 2007 and 2008). In the conclusive section of this article we will both identify and discuss some of the main problems that make difficult the psychological research in the paraguayan universities with special emphasis on the application of the experimental method.

Key words: Experimental Psychology, experimental method, psychology in Paraguay, psychological research.

\section{Introducción}

Por muy variados que puedan ser los enfoques teóricos de la Psicología contemporánea y por muy diversificados que resulten los diseños metodológicos utilizados en las investigaciones que los sostienen, hay un aspecto en el cual la mayoría de los psicólogos demuestran coincidencias, a pesar de la orientación conceptual que los represente: el visualizar a su disciplina como una actividad científica en forma primaria.

En efecto, tanto aquéllos que consagran su actividad cotidiana a los esfuerzos de producir conocimiento mediante el hallazgo de nuevas relaciones causales en el ambiente del laboratorio o quienes se hallan inmersos en el estudio de la realidad social, así como quienes traducen en forma diaria el lenguaje de la ciencia a la solución de situaciones prácticas que aparecen en la clínica, la escuela o la empresa, concuerdan al suponer que su disciplina no es la simple aplicación del sentido común a problemas de variada complejidad, sino la síntesis organizada de los diversos conceptos que emergen de la investigación de los más variados aspectos en el comportamiento humano y animal.

Al igual que su respeto por la autoridad de la ciencia, los psicólogos tienen muy asumida una identidad de origen. Esto se nota en que todos los libros enfocados a la historia de la Psicología proclaman el inicio de su etapa como disciplina científica, a partir de la fundación de un laboratorio en la ciudad alemana de Leipzig en 1879 por parte de Wilhelm Wundt (1832-1920), quien de este modo encarna a la perfección la imagen idealizada del científico que rescata a la Psicolo- 
gía de su vaguedad filosófica, para convertirla en una actividad con perfil de ciencia.

Pero el reconocimiento de este origen común de la Psicología no se refiere sólo al establecimiento del primer laboratorio en cuanto tal, sino que alcanza también a la adopción del método mismo. Es por esto que la Psicología Experimental aparece de continuo como un referente central en el escenario de las ciencias del comportamiento, mucho más cuando se la observa en una perspectiva histórica. Aunque también debe remarcarse su importancia para la Psicología del presente. Desde luego, esto no implica una descalificación hacia las demás metodologías existentes en la Psicología, que parten de postulados divergentes. Tampoco pretende situar al experimento y a la forma de interacción que éste presupone entre el investigador y su objeto de estudio, en una posición de absoluta superioridad epistemológica.

El punto de vista de un académico como Mandler (2007) es interesante a este respecto. Él culmina su historia de la Psicología Experimental realizando un paralelo con la Astronomía y la Paleobiología, dos disciplinas que han ganado un sitial de respetabilidad entre los más exigentes campos del conocimiento, pese a no ser experimentales. Podría pensarse en una reivindicación similar en el futuro para aquellos campos de la disciplina psicológica que no hacen uso activo del experimento. Aún así, es necesario reconocer que el método experimental se ha convertido en una suerte de icono en el marco de la Psicología Científica. En sentido general, la justificación epistemológica de éste se halla enclavada en las ciencias naturales (Corbetta, 2003) aunque su traslado al terreno de las ciencias sociales ha requerido adaptaciones fundamentales que se explican por la obvia diferencia entre sus objetos de estudio.

Según Alarcón (1991) si la Psicología Experimental es capaz de responder de mejor manera a la explicación de las causas a las que obedece la conducta, por lo que se considera su procedimiento como privilegiado frente a enfoques similares en ciencias sociales. Su confiabilidad como herramienta para el conocimiento se ha ganado debido al manejo activo y controlado de las variables que intervienen en la investigación y a su capacidad para encontrar respuestas claras a los interrogantes que surgen en el estudio del comportamiento. La metodología experimental permite influir en forma directa sobre las variables bajo análisis y establecer hipótesis causales claras y precisas (Hernández-Pina, 1998). Con frecuencia esta condición le ha valido una mayor proyección comparada a las otras metodologías de las que hace uso la Psicología.

En lo esencial, el método experimental es una forma de aplicación de la metodología científica a problemas que albergan la propiedad de ser resolubles (McGuigan, 1980) y se hallan claramente definidos (Pereda, 1987). Entre los investigadores del comportamiento se acepta por lo común que este es el método por excelencia en la ciencia (Arnau-Gras, 1992) y en verdad esta es la opinión mayoritaria, aunque debe reconocerse que no existe unanimidad plena al respecto (Rodríguez \& Pérez de Maldonado, 1995). Es significativo que muchos de quienes manifiestan disensos con respecto a la utilización del método experimental en la Psicología sean también quienes cuestionan el carácter científico de la misma.

Pero a pesar de controversias muchas veces marginales, la Psicología Experimental no ha cesado su ritmo de crecimiento y expansión constante durante todo el último siglo. Desde unos pocos tópicos iniciales en sus comienzos ha logrado abrirse hasta cubrir el espectro completo de la Psicología. Como apuntan Kantowitz, Roediger \& Elmes (2001), en la década de 1930 resultaba habitual que los investigadores experimentales se dedicaran al estudio de temas como la sensación y la percepción o el aprendizaje y la memoria, pero en la actualidad añadieron una variedad notable de puntos a su agenda investigadora, incluyendo la psicología ambiental, del desarrollo y de las diferencias individuales. Incluso áreas que en apariencia se encuentran muy distantes de los rigores necesarios en la experimentación, como es el caso de la Psicología Social, han asimilado sus recursos a la investigación del comportamiento en contextos colectivos (Insko \& Schopler, 1980). 
La incorporación del experimento al cuerpo metodológico principal de la Psicología justifica su presencia como área de estudio e investigación en los currículos de la mayoría de las carreras que existen en esta área. Esto se verifica también en países con una tradición científica menor, aunque la disponibilidad de recursos e infraestructura en muchos de ellos pueda variar de forma ostensible si se los compara con las naciones que disfrutan de un mayor peso en la producción psicológica global. Pese a ello, en algunos estados con desarrollo menor puede notarse un aporte pequeño aunque meritorio al conocimiento, aun con las dificultades que enfrentan para sostener la infraestructura que requiere la investigación de calidad.

En vista de lo mencionado, este artículo se propone analizar la producción actual en la cátedra de Psicología Experimental de dos instituciones paraguayas: a) La Universidad de la Integración de las Américas (UNIDA) que tiene funcionamiento en la ciudad de Asunción y b) la Universidad Católica Sede Regional Itapúa, que se encuentra en Encarnación. Ambos centros educativos cuentan con cátedras de Psicología Experimental que se imparten en sus carreras de Psicología. El trabajo parte de una breve panorámica sobre la enseñanza de la Psicología Experimental en el país para luego detenerse en la producción lograda en las dos instituciones señaladas durante los años 2006, 2007 y 2008. En la sección final se discuten los problemas principales que se enfrentan en la actualidad y las perspectivas futuras para el fortalecimiento de la disciplina.

\section{La Psicología Experimental en perspectiva}

Si tomamos en cuenta que las ideas nunca surgen de la nada sino de la suma o la síntesis de las aportaciones individuales, entonces no resultará extraño que la Psicología Experimental en cuanto concepto y campo de conocimiento demuestre antecedentes que se remontan a un tiempo anterior a su establecimiento formal en los claustros académicos. En Paraguay estos comienzos tomaron la forma de influencias intelectuales detectables en el pensamiento y los escritos de un grupo selecto de autores que dieron a conocer artículos y ensayos encuadrados en la Psicología, relacionándose a ella de forma indirecta o utilizando sus constructos para apoyar ideas en áreas más amplias.

Esto sucedía en los años iniciales del siglo XX.

A juzgar por la frecuencia de las menciones, la repercusión lograda por autores franceses como Théodule Ribot (1839-1916) pueden ubicarse en primer lugar, y la de alemanes como Wundt, en segundo término. Los trabajos que se registran en este período son importantes, no sólo por el carácter pionero que les corresponde para el desarrollo de una producción psicológica incipiente, sino también, porque los principios de una Psicología de corte científico se pueden localizar en ellos, en grados que son variables y se perciben de manera relativamente clara o ligeramente difusa, dependiendo de los propósitos y la profundidad que cada autor consiguió.

De los comentaristas de ideas se pasó a la enseñanza, por ello la fase siguiente coincidió con la creación de la primera cátedra de Psicología Experimental. El ámbito inicial fue externo a la universidad y correspondió al de una escuela normal de maestros, que por entonces eran los establecimientos destinados al entrenamiento de los profesionales que ejercían funciones en el magisterio de la república. Fue una de sus profesoras más destacadas, María Felicidad González (1884-1980), quien instituyó la primera cátedra en 1921, en forma conjunta con la apertura de la Escuela Normal de Profesores en Asunción. González ha merecido una apreciación destacada como una de las precursoras más importantes de la psicología paraguaya durante el período preuniversitario (García, 2006a, 2009c), principalmente por los textos que publicó (González, 1942, 1951) y por sus labores en la enseñanza. Más tarde fueron habilitadas otras cátedras similares en escuelas normales de diferentes puntos del país. Pero pese al llamado de personas dedicadas a la docencia como Juan Ramón Dahlquist (1884-1956) en favor de la apertura de laboratorios ya en los inicios del siglo XX (Dahlquist, 1912) no fue sino hasta octubre de 1959 que un recinto experimental pudo hacerse realidad en una institución educacional del país. 
El escenario fue la Escuela Normal de Profesores $N^{\circ} 1$ «Presidente Franco» en la zona céntrica de Asunción. El propulsor de este proyecto era el pedagogo ecuatoriano Emilio Uzcátegui, un erudito muy destacado en el ambiente educativo de su país y que por entonces se ubicaba al frente de la misión de UNESCO en Paraguay. El objetivo para este laboratorio fue introducir a los futuros profesores que cursaban sus estudios en la Escuela Normal al conocimiento y práctica de la Psicología Experimental (Uzcátegui, 1956). Aquí comprobamos de nuevo que el contexto para la aparición del primer laboratorio fue el de la educación, algo que resulta muy característico en la naciente Psicología Experimental paraguaya.

Pero las condiciones que acompañaron este emprendimiento no fueron las mejores que podían esperarse y el intento no prosperó. El creador del nuevo dispositivo debió abandonar el país muy poco después de su instalación debido al término de su misión oficial, y aunque se buscó entre los especialistas que se hallaban disponibles a alguien que pudiera actuar con solvencia para proseguir esta iniciativa, la decadencia fue inevitable y el laboratorio nunca tuvo la posibilidad real de producir fruto alguno (García, 2006a).

Cuando fueron instituidas las licenciaturas en Psicología durante la década de 1960, algunas clases de Metodología de la Investigación que estaban siendo dictadas permitieron la aplicación del método experimental a proyectos de investigación sencillos e incluso se realizaron trabajos utilizando sujetos animales. Éstos fueron dirigidos por Óscar Serafini en la Universidad Nacional de Asunción (UNA) a principios de la década de 1970. La cátedra en mención hizo uso del laboratorio de la Facultad de Ciencias Básicas de esta misma universidad como escenario para la implementación de sus experiencias. Pero no se mantuvieron por mucho.

La cátedra de Psicología Experimental aparece en 1985 integrando la carrera de Psicología de la Universidad Católica de Asunción. Ese año se dictó un seminario que en sus primeros dos semestres de aplicación tuvo carácter optativo y estuvo a cargo de John Marshall Throne, un académico de nacionalidad norteamericana afiliado a la Univer- sidad de Kansas en Lawrence. En este curso no se realizaron experimentos originales ni ejercicios de replicación sobre trabajos publicados. Las clases giraban en torno al estudio intensivo de la teoría del aprendizaje, principalmente del condicionamiento operante, con una fuerte tendencia hacia las aplicaciones a la educación especial. Pero Throne estuvo sólo un año enseñando en la Universidad Católica.

Entre 1986 y 1987 ejercieron la cátedra los psicólogos puertorriqueños Álvaro Pardo y María Moji$\mathrm{ca}$, quienes le imprimieron un mayor énfasis metodológico. A partir de allí comenzó a trabajarse con el desarrollo de investigaciones de los alumnos con la aplicación del método experimental a problemas de diversos tipos. La culminación de estos trabajos constituía un requisito obligatorio para la aprobación de la materia. Uno de los trabajos producidos en 1986 llegó incluso a la fase de publicación en una revista internacional de psicología (Elías, Mojica, Pardo \& Scappini, 1988). La influencia de estos profesores en el enfoque de la cátedra y en el estilo otorgado se ha dejado sentir también en otras instituciones incluso muchos años luego de su retiro (García, 2009a).

En 1989 fue abierta una carrera de Psicología en la filial de la UNA en Ciudad del Este, una comunidad que limita con el Brasil y se halla caracterizada por una actividad económica de primer orden. La institución pasó más tarde por un proceso de descentralización administrativa y académica para convertirse en Universidad Nacional del Este (UNE) (Ruíz-Carvallo, Medina, Godoy \& LaconichRomero, 1997).

En la misma ciudad, la Universidad Católica abrió otra carrera en 1994. Ambas tuvieron sus respectivas cátedras en el área, aunque en la UNE se la denominó «Psicología Experimental y Diseño de Proyectos». Se dictó por primera vez en 1996 pero tuvo una duración efímera, ya que después fue transformada simplemente en «Diseño de Proyectos». De este modo se eliminaba de su temario el estudio específico del método experimental. En cambio, la cátedra de la Universidad Católica se ha dictado en forma ininterrumpida desde 1996 hasta el presente. 
De las dos que se establecieron en la ciudad fue esta última la que aplicó de manera más productiva el modelo teoría-aplicación, dando lugar a una interesante cantidad de investigaciones concluidas. Su profesor hasta el 2002 fue un antiguo alumno de Pardo y Mojica. En los años siguientes aparecieron en Asunción otras cátedras similares repartidas en instituciones privadas como la Universidad del Cono Sur de las Américas (UCSA), que fundó en 1998 una carrera de Psicología Empresarial de cinco años y donde la cátedra que nos ocupa tiene un semestre de duración. La Universidad Americana (UA), que a la vez estableció su carrera de Psicología en el 2000 y que también se extiende por cinco años, dedica tres semestres a la enseñanza de la Psicología Experimental. Finalmente, la Universidad de la Integración de las Américas (UNIDA) que inició su opción académica en Psicología a comienzos del 2004, es la que dedica un período más prolongado al área de Psicología Experimental, otorgándole un total de cuatro semestres en su malla curricular. En esta última, la estructura de materias cubre nueve semestres. Una exposición más panorámica sobre la historia, características generales y la organización formal de varias de estas carreras puede encontrarse en el capítulo de García (2003a).

De forma coincidente la Universidad Católica impulsó el funcionamiento de tres nuevas carreras de Psicología en el año 2004 y con ello tuvieron su origen igual cantidad de cursos de Psicología Experimental en las sedes regionales de Concepción, Encarnación y Villarrica. En la actualidad se dispone de cuatro cátedras en Asunción, una en Ciudad del Este, una en Concepción, una en Encarnación y una en Villarrica. La mayoría de estas universidades combina una formación en las rutinas básicas de la metodología experimental con el desarrollo de pequeños proyectos de investigación que los estudiantes proponen a su elección y deben llevar a cabo durante el período lectivo. Estos son conducidos hasta una fase terminal para lograr la aprobación de la materia. La Universidad Nacional de Itapúa (UNI) en Encarnación también ha dictado seminarios de Psicología Experimental que no integran su estructura curricular fija en el 2004, 2007 y 2008. Desde luego, cada una de estas clases presenta aspectos de interés particular que merecerían un estudio individual y pormenorizado. Pero en lo que sigue de este reporte se expondrá en forma específica la producción concerniente a sólo dos de ellas.

\section{Fundamentación y método}

Conforme a lo expuesto previamente, en este artículo se aborda la producción en la cátedra de «Psicología Experimental IV» de la Universidad de la Integración de las Américas (UNIDA), una institución localizada en la ciudad de Asunción y «Psicología Experimental II» de la Universidad Católica «Nuestra Señora de la Asunción», Sede Regional Itapúa (UCl) en Encarnación. En la primera de las mencionadas, la materia cuenta con una duración de cuatro semestres (el tercero, el cuarto, el quinto y el sexto de los nueve que integran la carrera) y tiene como particularidad el mantener un eje temático particular en cada uno de ellos. Es así como Psicología Experimental I abarca los tópicos de la «Sensación y Percepción», Psicología Experimental II se refiere a «Motivación y Emoción», Psicología Experimental III comprende «Aprendizaje y Cognición» y Psicología Experimental IV está dirigida al «Pensamiento y Lenguaje».

La Universidad Católica contempla dos semestres para el abordaje de la Psicología Experimental (el quinto y el sexto de los doce en que está organizada su malla curricular). A diferencia de la UNIDA, sus dos partes no concentran estructuras temáticas específicas y el desarrollo presenta una orientación esencialmente metodológica. El método experimental se estudia con detalle en ambas etapas, pero es en la segunda en la que los estudiantes implementan una investigación propia que les sirve como requisito fundamental para la aprobación de la materia. De acuerdo con la modalidad que se impuso en la cátedra de Asunción desde su creación a mediados de la década de 1980 (García, 2009a) todas las sedes adoptan idéntico esquema, aunque con ligeras variaciones que no afectan la lógica esencial.

De esta manera, se asume que la producción lograda en dos universidades, que aunque perte- 
nezcan al mismo país reúnen perfiles muy diferenciados, ofrece una oportunidad inmejorable para comparar su producción y al mismo tiempo explorar las tendencias y direcciones que se puedan estimar dominantes en la investigación originada desde sus cátedras de Psicología Experimental. De las dos instituciones en estudio una es de capital privado y de reciente inauguración (la UNIDA) mientras la otra mantiene un carácter confesional al mismo tiempo que un perfil más tradicional y de mayor influencia en la sociedad paraguaya (la Universidad Católica).

La UNIDA se halla situada en la capital del país, Asunción, en tanto la Universidad Católica Sede Regional Itapúa se ubica en Encarnación, una antigua comarca que ostenta el rango de capital departamental. Ambas ciudades se hallan separadas a una distancia de 365 kilómetros. La veteranía de la Psicología en el ambiente académico de estas dos poblaciones tampoco es la misma. Como se ha mencionado en trabajos anteriores, la primera carrera de Psicología en Asunción se estableció en 1963, mientras que en Encarnación la opción inicial fue abierta en 1999 (García, 2009b).

La investigación adopta primariamente un enfoque descriptivo que toma como insumo básico los trabajos de investigación que fueron producidos por los alumnos de la carrera de Psicología, en la cátedra de «Psicología Experimental IV» de la UNIDA durante el segundo semestre del 2006 y en las clases de «Psicología Experimental II» de la Universidad Católica Sede Regional Itapúa durante el segundo semestre del 2006, el segundo semestre del 2007 y el segundo semestre del 2008. La clasificación de los escritos se realiza tomando en consideración los siguientes criterios:

a) El área al que corresponde la temática general de la investigación. Las categorías utilizadas son lo más generales posibles. Pero las clasificaciones propuestas no excluyen categorizaciones alternativas por lo que puede asignárseles un valor provisorio y no absoluto.

b) El grado de manipulación logrado con respecto a la variable independiente (VI) en cada trabajo, lo cual a su vez hace posible subdividirlas en: 1 )
Trabajos experimentales y 2) Trabajos cuasiexperimentales, siguiendo el conocido criterio de McGuigan (1980).

c) El grado de complejidad de la estadística que fuera puesta en práctica como recurso de análisis en las investigaciones consideradas.

Para quien precise una información más individualizada se ofrece en el Anexo 1 la lista completa de los trabajos comprendidos en este artículo, incluyendo sus respectivos títulos y autores.

\section{Resultados}

Investigaciones realizadas en la Universidad de la Integración de las Américas

Los trabajos elaborados por los estudiantes de «Psicología Experimental IV» de la Universidad de la Integración de las Américas en el año 2006 suman un total de cinco y pueden clasificarse de la siguiente manera:

\section{I. Área temática básica}

1. Aprendizaje de una segunda lengua: 1 trabajo. 2. Aprendizaje del lenguaje en el niño: 1 trabajo. 3. Desarrollo del pensamiento infantil: 1 trabajo. 4. Efectos cognitivos de la utilización de teléfonos celulares: 1 trabajo.

5. Embarazo durante la menopausia: 1 trabajo.

II. Diseño según el grado de manipulación de la variable independiente

1. Diseño experimental: 1.

2. Diseño cuasiexperimental: 3 .

3. Trabajo de carácter teórico: 1.

III. Tipo de estadística utilizada

1. Estadística descriptiva: 2.

2. Estadística inferencial: 0 .

3. No utiliza estadística: 3.

Investigaciones realizadas en la Universidad Católica, Sede Regional Itapúa

El segundo grupo de trabajos que integran esta revisión son los producidos por los estudiantes que cursan «Psicología Experimental II» en la Univer- 
sidad Católica, Sede Regional Itapúa, de la ciudad de Encarnación. En este caso se incluyen reportes de tres años, señalando primero los correspondientes al 2006 que suman en total ocho, luego los del año 2007 que comprenden igual número de investigaciones y finalmente los del 2008, que alcanzaron seis entregas. Éstas se agrupan haciendo uso de los mismos criterios utilizados en la clasificación anterior.

Año 2006

I. Área temática básica

1. Actitudes en relación con el sexo: 1 trabajo.

2. Aprendizaje de conductas agresivas en los niños: 1 trabajo.

3. Efectos cognitivos de la utilización de teléfonos celulares: 1 trabajo.

4. Influencias de la televisión y otros medios audiovisuales en la adquisición de comportamientos agresivos en niños y adolescentes: 2 trabajos. 5 . Ingesta de sustancias psicoactivas en adolescentes: 1 trabajo.

6. Psicología de la actividad física: 1 trabajo.

7. Psicología de la percepción: 1 trabajo.

II. Diseño según el grado de manipulación de la variable independiente

1. Diseño experimental: 3.

2. Diseño cuasiexperimental: 5 .

III. Tipo de estadística utilizada

1. Estadística descriptiva: 6 .

2. Estadística inferencial: 0 .

3. No utiliza estadística: 2 .

\section{Año 2007}

I. Área temática básica

1. Autovaloración y Depresión: 1 trabajo.

2. Comportamiento afectivo en niños de padres divorciados: 1 trabajo.

3. Efectos cognitivos de la estimulación temprana: 1 trabajo.

4. Estereotipos sociales y percepción de la belleza femenina: 1 trabajo.

5. Influencias de la violencia doméstica en la mujer: 1 trabajo.

6. Ingesta de alcohol y expresión gráfica: 1 trabajo.
7. Prejuicios hacia el divorcio en niños: 1 trabajo.

8. Estrés laboral: 1 trabajo.

II. Diseño según el grado de manipulación de la variable independiente

1. Diseño experimental: 3.

2. Diseño cuasiexperimental: 5 .

III. Tipo de estadística utilizada

1. Estadística descriptiva: 4.

2. Estadística inferencial: 0 .

3. No utiliza estadística: 4.

\section{Año 2008}

I. Área temática básica

1. Efectos psicológicos causados por el desarraigo: 1 trabajo.

2. Influencia de los estímulos verbales en el aprendizaje de niños pequeños: 1 trabajo.

3. Los juegos computarizados y la generación de conductas agresivas en los niños: 1 trabajo.

4. Memoria de palabras y género sexual: 1 trabajo. 5. Problemática causada por la reubicación de viviendas y establecimientos comerciales por la construcción de la hidroeléctrica de Yacyreta: 1 trabajo.

6. Utilización de la didáctica en el aprendizaje de la lectoescritura: 1 trabajo.

II. Diseño según el grado de manipulación de la variable independiente

1. Diseño experimental: 2.

2. Diseño cuasiexperimental: 3 .

3. Diseño cualitativo: 1.

III. Tipo de estadística utilizada

1. Estadística descriptiva: 4.

2. Estadística inferencial: 0 .

3. No utiliza estadística: 2 .

Principales dificultades

La Psicología Experimental paraguaya se ha desarrollado franqueada por la obvia paradoja de carecer de laboratorios. Sin embargo, esta insuficiencia tan importante no impidió el surgimiento de espacios de investigación alternativos que posibilitaron la implementación de algunos diseños sencillos. La estrategia principal para enfrentar 
la ausencia de laboratorios ha sido la adopción de condiciones exteriores lo más cercanas posible a las que se verifican en estos dispositivos, en una suerte de ampliación hacia el entorno social de las condiciones de previsibilidad y control que se asignan en los experimentos.

El que se hayan logrado o no estas metas a un grado satisfactorio es un problema que desde luego merece ser discutido con una amplitud mayor. Pero no debe pasarse por alto la importancia del producto final, es decir, la investigación aplicada a problemas que -en su gran mayoría- guardan una connotación social mucho más acentuada de lo que podría ser la regla con estudios de ciencia básica conducidos en un laboratorio normal. No obstante, el desempeño de la investigación en las condiciones mencionadas ha tropezado con algunos problemas muy específicos que caracterizan a la psicología experimental paraguaya. Podrá comprenderse que muchos de ellos tienen su origen en la reducida rutina investigadora que todavía afecta a la universidad como un todo y a sus carreras de psicología en particular, tal como ha sido subrayado en publicaciones previas que abordan los problemas para la formación del psicólogo en el país (García, 2003a, 2003b, 2009d).

En este amplio contexto, los problemas particulares en las cátedras de Psicología Experimental analizadas en este trabajo y que afectan a una importante proporción de los proyectos presentados cada año son los siguientes:

a. Reconocimiento de problemas relevantes y elaboración de hipótesis. Con frecuencia los proyectos de investigación presentados a las cátedras de Psicología Experimental adolecen de cierta oscuridad en lo que se refiere a la formulación de los problemas, y muchas veces esta dificultad persiste incluso más allá de las etapas iniciales en la elaboración del proyecto. Es el caso que algunos estudiantes, al llegar el momento de encarar su entrenamiento en el método experimental, demuestran limitaciones importantes para lograr una conceptualización correcta y en el análisis de la verdadera base y fundamento que subyace a la problematización de su investigación.
Esto los lleva a permanecer en un nivel de comprensión muy básico que no alcanza al problema real en toda su dimensión. Es frecuente que en los trabajos se planteen problemas que no guardan relación directa con el diseño metodológico a ser aplicado con posterioridad, o resulten muy limitados o muy amplios en su alcance. La dificultad para la formulación de buenas hipótesis es otro aspecto asociado. Aquí el rol del lenguaje resulta decisivo. La utilización de un vocabulario concreto y que al mismo tiempo resulte lo suficientemente descriptivo son los escollos más comunes. También es necesario incentivar al alumno a formular hipótesis que sean científicamente relevantes y que se proyecten más allá de la obviedad que suponen los dictados del sentido común para que puedan representar un aporte con cierto grado de originalidad o novedad.

b. Elección de la técnica y procedimiento adecuado. El método experimental, en cuanto tal, mantiene unas condiciones y características que son bien identificables en sus normas procedimentales básicas y que lo diferencian claramente de otros que son utilizados por la Psicología. Aún así, los alumnos tropiezan con la dificultad para escoger una técnica que se ajuste de manera efectiva a los lineamientos básicos exigidos por el experimento, y muchas veces se desencadena una sustitución por otras estrategias como la aplicación de tests psicométricos en la metodología correlacional, la entrevista o la simple observación del comportamiento, que resultan muy válidas en otros contextos, pero que no se pueden caracterizar plenamente como experimentales.

El aspecto más malo en estos casos de sustitución de un método por otro es el control experimental (McGuigan, 1980), una condición absolutamente esencial para esta metodología en particular, debido al manejo privativo de las variables que su aplicación estricta permite. El plantear un diseño de tipo experimental utilizando técnicas que no son apropiadas a sus necesidades de rigor acaba en una asociación híbrida que no satisface plenamente un manejo riguroso de la causalidad comportamental. 
c. Eficiente organización del tiempo. Una queja común que manifiestan los estudiantes es la escasa disponibilidad de tiempo para materializar sus proyectos de investigación. Éste, desde luego, es más un problema atinente a los hábitos y la disciplina de los propios alumnos, la circunstancia particular en que cada uno vive y su capacidad de organización personal y no merecería recogerse como una característica principal, excepto por un detalle importante: el perfil más convencional que asumen las carreras de Psicología en el Paraguay corresponde a aquéllos roles del psicólogo más direccionados a las aplicaciones profesionales y mucho menos a las posibilidades de este profesional como un agente activo en la producción de conocimiento científico. Algunas instituciones, incluso, han eliminado la presentación de tesis como requisito final para el egreso o las han vuelto optativas. Todo esto hace que se asigne un espacio comparativamente mayor a las tareas relacionadas con la práctica, las pasantías y las materias profesionales que a la investigación propiamente dicha. El tiempo dedicado a materias como Psicología Experimental casi siempre sufre los recortes introducidos por los propios alumnos en el ordenamiento de sus prioridades académicas, relegando las tareas de planificación, búsqueda bibliográfica y diseño de experimentos a una agenda accesoria al resto de sus actividades como alumno.

d. Escaso soporte bibliográfico. Los órganos primarios de difusión de los avances en la psicología experimental moderna son las revistas especializadas. Sin ellas, resulta muy difícil tener un acceso a las fuentes más autorizadas y completas de la información. Un sustituto que puede ser aprovechado dentro de límites muy precisos es Internet, donde algunos artículos que fueron publicados previamente en revistas son cargados a la red en sus formatos originales.

En la actualidad, con la puesta en funcionamiento de sistemas de archivo como Scielo, que ya ha desarrollado colecciones muy completas para algunos países, o las bases de datos en crecimiento como Redalyc o Dialnet, muchos textos de Psicología han comenzado a volverse más accesibles. Pero aún dejan un margen relativamente limitado para una investigación sistemática. Como ha sido señalado en trabajos anteriores (García, 2003a, 2003b), todavía es poco común que las universidades paraguayas cuenten con secciones de revistas de psicología. Las dos instituciones a que nos hemos referido en este artículo se cuentan entre aquéllas que no las poseen.

Así, una revisión bibliográfica bien informada para sostener las investigaciones de los alumnos reposa casi con exclusividad en el material que se encuentra disponible en Internet. Con las ventajas, pero también con todas las limitaciones que esta única fuente de acceso a las publicaciones originales puede suponer para realizar trabajos correctamente fundamentados en su esquema teórico.

e. Ausencia de investigaciones previas. Muy relacionado con el punto anterior está el problema de la ausencia de investigaciones previas en el nivel nacional respecto a la mayoría de los problemas que son posibles de estudio en una cátedra de Psicología Experimental. Los estudiantes no cuentan con archivos organizados donde puedan acceder a un registro acumulativo sobre las investigaciones realizadas con anterioridad. Tampoco es frecuente que se disponga de órganos de sistematización de la información. Una excepción muy meritoria es la revista Eureka que la cátedra de Psicología Experimental de la Universidad Católica de Asunción ha puesto en circulación y que consiguió publicar cuatro números hasta ahora. En ellos se compendian varios de los trabajos generados desde esa instancia académica en los últimos años. La revista, sin embargo, tiene el inconveniente de ser muy irregular y con una política de distribución de corto alcance que necesita ser mejorada para lograr un impacto mayor. No obstante, si otras instituciones optaran por una senda editorial similar es posible que en el mediano plazo se pudiera acceder a una base de datos de contenido más general y sistematizado.

f. Laboratorios. En varias partes de este trabajo se ha mencionado que la enseñanza en el método experimental debe realizarse sin la disponibilidad efectiva de laboratorios. El problema, sin embargo, se ve ampliamente reforzado por una cuestión 
de imagen que afecta a las carreras de Psicología en el Paraguay, tanto en el ámbito de las instancias directivas como de muchos estudiantes. En efecto, aquí la disciplina es vista como una opción de inversión barata en el marco de ejecución de los presupuestos académicos y ha sido, predominantemente, una carrera de pizarrón y tiza (García, 2003a). Refractarios a incurrir en inversiones que supongan lo que ellos perciben como gastos onerosos, las burocracias universitarias se han mostrado renuentes a cualquier pedido de instalar dispositivos experimentales en sus carreras de Psicología. Lo que también se ha constatado es la escasa efectividad demostrada por los mismos psicólogos para constituirse en publicistas adecuados sobre los requerimientos que presenta un tipo de investigación más exigente desde el punto de vista metodológico. Por lo que esta necesidad de crear una conciencia pública bien informada es otro aspecto que debería ser trabajado en el corto plazo.

g. El personal docente. En Paraguay existen pocas personas con el entrenamiento, la experiencia y el interés suficientes para dedicarse con verdadera efectividad a la enseñanza de la psicología experimental y encarar la dirección de trabajos de investigación. En varios puntos geográficos que se hallan a grandes distancias de la capital Asunción, la necesidad de personal idóneo es incluso más acuciante. Es en esta ciudad donde se halla concentrada la mayor cantidad de psicólogos y el lugar en que residen los que cuentan con una formación especializada. Esto provoca que unos pocos buenos conocedores del método experimental se encuentren absorbidos por varias instituciones al mismo tiempo e incluso deban trasladarse a otras regiones del país para dictar clases.

También existe el problema que, bajo la presión de nombrar profesores al frente de sus cátedras, en muchas de las carreras que se hallan en puntos distantes y aún en algunas de la capital resulte forzoso convocar a personas sin experiencia en la investigación o con un aprendizaje poco profundo del método. Al mismo tiempo, quienes se encargan de la contratación de los profesores no siempre proceden apoyados en mecanismos objetivos -como concursos de cátedra bien organizados- y por ello no es extraño que incurran en elecciones deficientes, muchas veces presididas por relaciones de carácter personal en sustitución de criterios más institucionales. Por lo que asegurar la mejor selección del personal docente es otro de los puntos a ser superados todavía.

h. Ausencia de unidades y programas de investigación consistentes. El trabajo de investigación que tiene lugar en los cursos de Psicología Experimental de las dos universidades mencionadas se mantiene exclusivamente como una iniciativa de las materias respectivas, pero con reducido acompañamiento institucional. Es lamentable reconocer que en Paraguay esta desconexión entre la cátedra y la entidad anfitriona es frecuente. Aún en los pocos casos en que existen instancias de coordinación de investigaciones en las facultades y las carreras de Psicología, pueden observarse interacciones muy discontinuas entre estos departamentos y las cátedras individualmente consideradas. Ciertamente, la UNIDA cuenta con un Núcleo de Investigación y Posgrado, pero su énfasis parece más dirigido a los trabajos generados en los diferentes cursos posgraduados que la universidad ofrece y bastante menos hacia las materias individuales que componen las diferentes carreras. Desde luego, la existencia de políticas institucionales que asignen líneas maestras o prioridades especiales para la investigación es de ausencia todavía más notoria. Por tanto, los esfuerzos creativos que se llevan a cabo desde ambas cátedras de Psicología Experimental no sólo están muy privados de los sustentos institucionales mencionados, sino a la vez muy desregulados y sujetos en demasía a las iniciativas de los estudiantes. Éstas, por ser coyunturales en esencia, requieren de algún marco regulador general que actúe, por lo menos, al grado mínimo de sugerir posibles proyectos de relevancia y que, al mismo tiempo, sea capaz de proveer la infraestructura básica sin la cual es casi imposible un trabajo científico de calidad.

i. Habilidades para la utilización de un lenguaje apropiado para la redacción de artículos. Enseñar a escribir con la parsimonia y la precisión conceptual que son exigidas en la ciencia moderna, es una de las tareas más difíciles que puedan aco- 
meterse. Es únicamente bajo la disciplina de una práctica repetida que el estudiante puede adquirir la versatilidad necesaria en el lenguaje propio de la investigación, pero aún así el ejercicio no siempre es suficiente. En la actualidad es de uso rutinario el texto que la American Psychological Association publica con el nombre de Manual de Publicaciones (American Psychological Association, 2001) y que establece pautas comunes en aspectos relacionados con la organización y redacción de los artículos científicos para la mayor parte de las revistas del mundo. Existen otros materiales muy útiles para la adquisición de estas pericias básicas (Nicol \& Pexman, 1999, Sternberg, 1996).

Las dos cátedras de Psicología Experimental referidas adoptan este material como elemento básico para la redacción de los informes finales de los alumnos. Por lo que un entrenamiento mínimo en las habilidades de redacción constituye una tarea adicional que debe fijarse el docente. Haciendo una revisión de los productos que estas cátedras han venido lanzando puede comprobarse con facilidad que las destrezas para escribir con precisión, concisión y pertinencia se alcanzan -en la amplia mayoría de los casos- en grados que posiblemente resultan inferiores a los que de ordinario se requieren en las revistas especializadas. Esta circunstancia podría explicar también que de las publicaciones con temática psicológica logradas por autores locales en periódicos de circulación internacional (García, 2006b) solo figure un trabajo proveniente de una cátedra de psicología experimental y esto en 1988, hace ya más de veinte años.

j. Falta de una comprensión adecuada de la naturaleza de la investigación. En países donde la investigación científica resulta escasa es común enseñar las reglas de la metodología como normas duras y estrictas en lugar de infundir las actitudes cognitivas necesarias para abrirse sin prejuicios y con amplitud a los problemas que caben en la agenda de la ciencia. La curiosidad intelectual se estimula por una permanente sorpresa ante los problemas que depara la realidad y por la experiencia de una pregunta inteligente a las explicaciones ofrecidas por las teorías en vigen- cia. El cuestionamiento útil presupone siempre una comprensión ordenada de la naturaleza de los fenómenos y una actitud abierta y antidogmática. Pero también requiere información suficiente, esto es, conocimiento organizado y solvente en relación con el estado de avance en función de un problema determinado.

Debido a las características en las que ha tenido que desenvolverse la enseñanza de la Psicología Experimental en el Paraguay, muchas de estas condiciones son difíciles de obtener. Con frecuencia los estudiantes se muestran excesivamente dependientes de los consejos del profesor en la elección de sus temas de investigación o se acobardan con facilidad ante los múltiples obstáculos existentes, en especial, la carencia de infraestructura y de buena y actualizada bibliografía. De esta manera, acaban escogiendo aquellos temas que les sugieren un menor trabajo. La simple replicación de experimentos ya conocidos y confirmados es una tentación permanente, con lo cual se esquiva de manera muy directa el desafío que supone el pensamiento original. Se cae muchas veces en la postulación pura y simple de hipótesis que resaltan por su obviedad, convirtiendo el proceso de investigación en la búsqueda de hallazgos enteramente previsibles.

Como consecuencia, la investigación muchas veces se transforma en una tarea realizada por mero compromiso ante la ausencia de motivaciones inquisitivas reales. Existe más un temor ante el fracaso que una decisión real de poner a prueba supuestos propios. Por ello, la actitud característica del verdadero investigador, que consiste en arriesgarse en la formulación de hipótesis y diseños capases de ubicarlo en el camino del verdadero descubrimiento científico, es una de las virtudes que con menor fuerza se encuentran en la mayor parte de los estudiantes de Psicología.

\section{Conclusión}

Los retos que enfrenta el avance de la psicología experimental en el Paraguay de cara a su afianzamiento efectivo son numerosos y complejos. En buena medida, estas dificultades son las mismas que afectan a la investigación psicológica en ge- 
neral, pero tienen algunas características que son específicas. En cuanto concepto, sin embargo, la psicología que se apoya en la aplicación del método experimental tiene una historia reconocible al menos desde 1887. Como disciplina universitaria tiene una inserción indirecta en los programas académicos desde inicios de la década de los sesenta y una presencia concreta desde los años ochenta. Hoy se cuenta con clases de psicología experimental en ocho universidades paraguayas donde todas, sin embargo, adolecen de limitaciones similares.

Lo importante es que, pese a las falencias, algunas de estas cátedras han logrado avanzar hasta el estadio que supone la conclusión de trabajos de investigación, que además de cumplir con la función didáctica de aprender el manejo de la metodología, son una fuente potencial de conocimiento. Puede estimarse como un compromiso pendiente para los años venideros el lograr un crecimiento cualitativo que permita el ingreso a una etapa de mayor proyección hacia la psicología latinoamericana y universal. Para el logro de esta meta será necesario aportar ideas, conceptos y teorías nuevas que hagan posible implementar líneas de investigación independientes. También habrá que potenciar un desplazamiento en el rol del docente como agente divulgador de conocimientos al de investigador activo, transformando lo que hoy son ejercicios de replicación de cátedra en ejemplos auténticos de innovación creativa. Después de todo, la ciencia auténtica camina lejos de la repetición rutinaria y muy próxima a la novedad y el descubrimiento genuinos.

\section{Referencias}

Alarcón, R. (1991). Métodos y diseños de investigación del comportamiento. Lima: Universidad Peruana Cayetano Heredia, Fondo Editorial.

American Psychological Association. (2001). Manual of the American Psychological Association, (5a. ed.). Washington D.C.: American Psychological Association.

Arnau-Gras, J. (1992). Psicología experimental. Un enfoque metodológico. México: Trillas.
Corbetta, P. (2003). Metodología y técnicas de investigación social. Madrid: McGraw Hill.

Dahlquist, J. R. (1912). Páginas de un maestro. Asunción: Talleres Tipográficos del Estado.

Elías, R., Mojica, M., Pardo, A. \& Scappini, L. (1988). Delincuencia juvenil y juicio social. Revista Latinoamericana de Psicología, 20, 217-224.

García, J. E. (2003a). Problemas centrales en la formación académica y el entrenamiento profesional del psicólogo en el Paraguay. En Villegas, J. F., Marassi L. P. \& Toro C., J. P. (Eds.). Problemas centrales en la formación académica y el entrenamiento profesional del psicólogo en las Américas, Vol. II (pp. 205-279). Santiago: Sociedad Interamericana de Psicología.

García, J. E. (2003b). Desafíos para el afianzamiento de la investigación psicológica en el Paraguay. Estudos e Pesquisas em Psicologia, 3 (2), 11-36.

García, J. E. (2006a). Relaciones históricas entre la Psicología y la educación en Paraguay. Psicologia da Educaçâo, 22, 95-137.

García, J. E. (2006b). Publicaciones paraguayas en el área de la Psicología: 1960-2005. Revista Latinoamericana de Psicología, 38 (1), 149-167.

García, J. E. (2009a). La cátedra de Psicología Experimental en la Universidad Católica de Asunción: 1985-1987. En prensa.

García, J. E. (2009b). Historia y estado actual de la Psicología en la ciudad de Encarnación, Paraguay. En prensa.

García, J. E. (2009c). Breve historia de la Psicología en el Paraguay. Psicología para América Latina, 17. Recuperado el día 12 de abril de 2010 de: http://psicolatina.org/17/paraguay. html. 
García, J. E. (2009d). La enseñanza de la Psicología en la Universidad Nacional de Asunción: estudio de nueve materias prototípicas. En prensa.

González, M. F. (1942). Misceláneas paidológicas para padres y educadores. Asunción: Edición de la autora.

González, M. F. (1951). Organización escolar. Manual de Pedagogía fundamental para estudiantes de los cursos normales ( $2^{\mathrm{a}}$. ed.). Asunción: La Colmena.

Hernández-Pina, F. (1998). La investigación experimental. En Buendia Eisman, L., Colás Bravo, P. \& Hernández Pina, F. (Eds.), Métodos de investigación en psicopedagogía (pp. 61-90). Madrid: McGraw-Hill.

Insko, C. A. y Schopler, J. (1980). Psicología social experimental. México: Trillas.

Kantowitz, B. H., Roediger, H. L. \& Elmes, D. G. (2001). Psicología Experimental. Cómo entender las investigaciones psicológicas. México D.F.: Thomson Learning.

Mandler, G. (2007). A history of modern experimental psychology. From James and Wundt to cognitive science. Cambridge, M.A.: Bradford Books/The MIT Press.
McGuigan, F. J. (1980). Psicología Experimental. Enfoque metodológico. México: Trillas.

Nicol, A. M. \& Pexman, P. M. (1999). Presenting your findings. A practical guide for creating tables. Washington D. C.: American Psychological Association.

Pereda, S. (1987). Psicología Experimental I. Metodología. Madrid: Pirámide.

Rodríguez, A. \& Pérez de Maldonado, I. (1995). La investigación experimental en ciencias sociales. México: Trillas.

Ruíz-Carvallo, G., Medina, R. E., Godoy, J. F. \& Laconich-Romero, A. (1997). Por sus frutos los conoceréis. Antecedentes, creación y primeros pasos de la U.N.E. Ciudad del Este: Universidad Nacional del Este.

Sternberg, R. J. (1996). Investigar en Psicología. Una guía para la elaboración de textos científicos dirigida a estudiantes, investigadores y profesionales. Barcelona: Paidós.

Uzcátegui, E. (1956). Establecimiento de un gabinete de Psicología y Psicopedagogía en la Escuela Normal de Profesores $\mathrm{N}^{\circ} 1$ de Asunción. Boletín de Educación Paraguaya, 1 (3), 40-41. 


\section{Anexo 1. \\ Lista de investigaciones producidas en la cátedra de «Psicología Experimental IV» de la Universidad de la Integración de las Américas (UNIDA) durante el año 2006 y la Universidad Católica Sede Regional Itapúa (UCl) durante los años 2006, 2007 y 2008}

\section{Universidad de la Integración de las Américas (UNIDA), año 2006}

1. Armoa, Rosa; Ruiz, Shirley; Cáceres, Verónica y Bordón, Isabel: Relación de escritura en celular y calidad de la ortografía.

2. Asad, Nadia; Bordón, Noemí; Tellechea, María de la Paz y Villalba, Rita: Influencia del habla dirigida al niño en el lenguaje preescolar.

3. Cáceres, Laura; Kassar do Nascimento, Janaina; Cardozo, Vera y Doldán, Rossana: ¿Qué es el pensamiento para los niños?

4. Faustino, Joralvo: Embarazo en la menopausia.

5. Monteiro, Chevonne; Giménez, Liz y Bareiro, Marlene: Aprendizaje y escritura de un segundo idioma.

\section{Universidad Católica, Sede Regional Itapúa (UCI), año 2006}

1. Bado Méndez, Melisa Raquel: Influencias de la TV en el niño.

2. Berestovoy, Natalia; Fuentes, Patricia; Trinidad, Avelina y Chaparro, Raquel: Uso de los teléfonos celulares y la calidad de ortografía.

3. Blaich, Daisy y Utz, María Vanesa: Influencias de estímulos audiovisuales violentos en niños y adolescentes.

4. Duba, Yamil; Sozaki, Yumi; Shimanaka, Fumi y Szopa, Analía: Recreación del "change blindness" motivado por la técnica de parpadeo (flicker technique) en alumnos de la Universidad Católica Sede Regional Itapúa.

5. Escobar B., Claudia Leticia; Núñez R., Claudia Analía; Duarte N., Clara Isabel y Benítez O., Lourdes Ester: Uso de drogas en una muestra de adolescentes.

6. Insfrán Novisky, María Leticia; Paredes Ledesma, Sirley Noelia; Ramírez López, Alicia Antonia y Zapata Rodríguez, Paola Adriana: Sedentarismo, Depresión y Actividad Física.

7. Mallorquin, Dina; Cuevas de Garay, Liliana y Villalba Villalba, Hugo: Agresividad en niños y niñas de 5 a 11 años.

8. Van Heugten, María Christina; Mayeregger, Carmen y Urtlauf, Melisa: Actitud de los adolescentes ante las ETS y el sexo en general.

\section{Universidad Católica, Sede Regional Itapúa (UCI), año 2007}

1. Báez, Diana; Senem, Ligia y Hilscher, Cindy: Estrés laboral en cajeros bancarios y municipales.

2. Domínguez, Noelia; González, Kathya y Mereles, Adriana: Impacto de la violencia doméstica en la salud de la mujer maltratada.

3. Gamarra Silva, Analía y Vera Krug, Perla: Influencias de la estimulación temprana sobre la cognición en niños.

4. Haurón Rosner, María del Carmen; Talavera Tank, Elsa Yissel y Villalba Rivas, Andrea Elizabeth: Comparación de la expresión gráfica de personas con problemas de adicción al alcohol y las no alcohólicas.

5. Lezcano, Danna; González, Evangelina y González, Lilia: Estereotipos de la belleza femenina.

6. Meza, Cecilia y Fretes, Andrea: El divorcio, un problema que afecta a la sociedad.

7. Regis, Roberto Daniel y Romero, Raquel: Efectos del divorcio de los padres en la conducta afectiva y cognitiva de los hijos.

8. Villalba, Celeste y Ortellado, Tania: La autovaloración ayuda a determinar que los adolescentes poseen un estado depresivo. 


\section{Universidad Católica, Sede Regional Itapúa (UCl), año 2008}

1. Barbieri Hamann, Verónica y Montiel González, Ada: La utilización de la didáctica en las aulas da resultados muy favorables.

2. Bottger, Cynthia y Rivas, Mirian: Mejores respuestas memorísticas de palabras presentadas en función al sexo.

3. Duarte, Yolanda Maribel y Martínez, Elba: Efecto de los estímulos verbales positivos y los reforzadores como motivación en el aprendizaje de los niños.

4. Esteche, Karina y Semenaka, Diana: Juego computarizado, conductas agresivas en niños.

5. Jarenko, Natalia y Chamorro, Diana: El desarraigo afecta el relacionamiento.

6. Millán, Paola y Androsczuk, Lidia: Problemática de la reubicación. 